\title{
Block Empirical Likelihood for Semiparametric Varying-Coefficient Partially Linear Errors-in-Variables Models with Longitudinal Data
}

\author{
Yafeng Xia and Hu Da \\ School of Science, Lanzhou University of Technology, Lanzhou, Gansu 730050, China \\ Correspondence should be addressed to Yafeng Xia; gsxyf@aliyun.com
}

Received 8 April 2013; Revised 29 September 2013; Accepted 29 September 2013

Academic Editor: Shein-chung Chow

Copyright (c) 2013 Y. Xia and H. Da. This is an open access article distributed under the Creative Commons Attribution License, which permits unrestricted use, distribution, and reproduction in any medium, provided the original work is properly cited.

Block empirical likelihood inference for semiparametric varying-coeffcient partially linear errors-in-variables models with longitudinal data is investigated. We apply the block empirical likelihood procedure to accommodate the within-group correlation of the longitudinal data. The block empirical log-likelihood ratio statistic for the parametric component is suggested. And the nonparametric version of Wilk's theorem is derived under mild conditions. Simulations are carried out to access the performance of the proposed procedure.

\section{Introduction}

For longitudinal data, we consider semiparametric varyingcoefficient partially linear model which has the following form:

$$
Y(t)=X(t)^{\tau} \beta+Z(t)^{\tau} \theta(t)+\epsilon(t),
$$

where $Y(t)$ is the response variable, $X, Z$, and $t$ are regressors, $\beta=\left(\beta_{1}, \ldots, \beta_{p}\right)^{\tau}$ is a $p$-dimensional vector of unknown parameters, $\theta(t)=\left(\theta_{1}(t), \ldots, \theta_{q}(t)\right)^{\tau}$ is a $q$-dimensional vector of smooth functions of time $t$, and $\epsilon(t)$ is a zero-mean stochastic process. Due to the curse of dimensionality, for simplicity, we assume that $t$ is univariate.

Obviously, model (1) contains many usual parametric, nonparametric, and semiparametric models. Model (1) has been studied by many authors. Zhang et al. [1] suggested a two-step method for estimating it. Li et al. [2] suggested a local least-squares procedure with a kernel weight function. Fan and Huang [3] developed a profile least-squares technique for estimating parametric model . You and Zhou [4] and Huang and Zhang [5] suggested the estimator of the parametric and nonparametric models, respectively. Fan et al. [6] proposed a semiparametric estimation of the working correlation matrix and applied a profile weighted leastsquares approach.

However, in many practical situations, these variables are often measured with error. In this paper, we consider this case where the variable $X(t)$ is measured with additive error and both $Z(t)$ and $t$ are measured exactly. That is, $X(t)$ cannot be observed, but an unbiased measure of $X(t)$, denoted by $W(t)$, can be obtained as follows:

$$
W(t)=X(t)+U(t)
$$

where $U(t)$ is the measurement error, which is independent of $\left(X^{\tau}(t), Z^{\tau}(t), \epsilon(t), t\right)$, with mean zero and covariance matrix $\Sigma_{u u}$. We can assume that $\Sigma_{u u}$ is known. If $\Sigma_{u u}$ is unknown, we estimate it by repeatedly measuring $W(t)$ by Liang et al. [7]. For errors-in-variables models (1) and (2), Liang et al. [8] developed a profile least-squares procedure to estimate the parametric component and derived the asymptotic normality of the resulting estimator.

The empirical likelihood, which is a nonparametric approach for constructing confidence regions, was introduced by Owen [9] and has many nice statistical properties (see Owen [10]). Owen [11] applied empirical likelihood to linear regression models and Kolaczyk [12] made further extensions to generalized linear models. Recently, Xue and 
Zhu [13] considered the varying coefficient models. You and Zhou [4], Huang and Zhang [5], and Zhao and Xue [14] investigated the empirical likelihood confidence regions for varying-coefficient partially linear models. Other related papers contain Yang and Li [15], Hu et al. [16], Wang et al. [17], and Fan et al. [18, 19].

In this paper, we consider models (1) and (2) with longitudinal data; one aim of this paper is to construct the confidence region for the parameter components. To achieve it, we apply the block empirical likelihood approach [20] to construct block empirical log-likelihood ratio statistic for parameter $\beta$ and then prove nonparametric Wilk's phenomenon. Simulation studies assess the proposed method. The other aims are to prove that the maximum empirical likelihood estimator (MELE) for the parameter is asymptotically normal under some suitable conditions.

The rest of this paper is organized as follows. In Section 2, we construct the block empirical likelihood based confidence region for the parametric components. Assumption conditions and main results are given in Section 3. Simulation results are reported in Section 4. The proofs of the main results are stated in Section 5. Finally, some concluding remarks are given.

\section{Methodology}

In this section, we are to extend the result of $\mathrm{Hu}$ [21] to the semivarying coefficient errors-in-variables model with longitudinal data.

We apply longitudinal data $\left(Y_{i}\left(t_{i j}\right), X_{i}\left(t_{i j}\right), Z_{i}\left(t_{i j}\right), t_{i j}\right)$. $i=1, \ldots, n$, and $j=1, \ldots, n_{i}$ which are generated from semivarying coefficient errors-in-variables model through the following equation:

$$
\begin{gathered}
Y_{i j}=X_{i j}^{\tau} \beta+Z_{i j}^{\tau} \theta\left(t_{i j}\right)+\epsilon_{i j}, \\
W_{i j}=X_{i j}+U_{i j},
\end{gathered}
$$

where $Y_{i j}=Y_{i}\left(t_{i j}\right), X_{i j}=X_{i}\left(t_{i j}\right), Z_{i j}=Z_{i}\left(t_{i j}\right)$ and $\epsilon_{i j}=\epsilon_{i}\left(t_{i j}\right)$, and $U_{i j}=U_{i}\left(t_{i j}\right), i=1, \ldots, n, j=1, \ldots, n_{i}$. We use counting process $N_{i}(t) \equiv \sum_{j=1}^{n_{i}} I\left(t_{i j} \leq t\right)$ to describe the number of observations of the $i$ th subject. We assume that $n_{i}$ is bounded, but the number of subjects $n$ goes to infinity.

Suppose that $\beta$ is known; then, model (3) can be reduced to a varying-coefficient regression model:

$$
\begin{array}{r}
Y_{i j}-X_{i j}^{\tau} \beta=\sum_{k=1}^{q} Z_{i j k} \theta_{k}\left(t_{i j}\right)+\epsilon_{i j}, \\
i=1, \ldots, n, \quad j=1, \ldots, n_{i} .
\end{array}
$$

Here, the local linear regression method is applied to estimate the coefficient function $\left\{\theta_{j}(\cdot), j=1, \ldots, q\right\}$ in model (4). That is, for $t$ in a small neighborhood of $t_{0}$, one can approximate $\theta_{j}(\cdot)$ locally by a linear function

$$
\begin{aligned}
\theta_{j}(t) & \approx \theta_{j}\left(t_{0}\right)+\theta_{j}^{\prime}\left(t_{0}\right)\left(t-t_{0}\right) \\
& \equiv a_{j}+b_{j}\left(t-t_{0}\right), \quad j=1, \ldots, q,
\end{aligned}
$$

where $\theta_{j}^{\prime}(t)=\partial \theta_{j}(t) / \partial t$. This leads to the following weighted least-squares problem: find $\left\{\left(a_{j}, b_{j}\right), j=1, \ldots, q\right\}$ to minimize

$$
\begin{aligned}
\sum_{i=1}^{n} \int_{0}^{1} & \left\{Y_{i}(s)-X_{i}^{\tau}(s) \beta-\sum_{k=1}^{q}\left\{a_{k}+b_{k}(s-t)\right\} Z_{i k}(s)\right\}^{2} \\
& \times K_{h}(s-t) d N_{i}(s)
\end{aligned}
$$

where $K$ is a kernel function, $K_{h}(\cdot)=K(\cdot / h) / h$, and $h$ is a bandwidth. Let

$$
\begin{aligned}
& \Omega_{t} \operatorname{diag}\left(K_{h}\left(t_{11}-t\right), \ldots, K_{h}\left(t_{1 n_{1}}-t\right), \ldots, K_{h}\left(t_{n n_{n}}-t\right)\right) \\
& Y=\left(Y_{1}\left(t_{11}\right), \ldots, Y_{1}\left(t_{n 1}\right), \ldots, Y_{n}\left(t_{n n}\right)\right)^{\tau}, \\
& X=\left(X_{1}^{\tau}, \ldots, X_{n}^{\tau}\right)^{\tau}, \\
& Z=\left(\begin{array}{ccc}
Z_{11}^{\tau} \\
\vdots \\
Z_{1 n_{1}}^{\tau} \\
\vdots \\
Z_{n n_{n}}^{\tau}
\end{array}\right) \\
&=\left(\begin{array}{ccc}
Z_{1}\left(t_{11}\right) & \ldots & Z_{1}\left(t_{1 q}\right) \\
\vdots & \ddots & \vdots \\
Z_{1}\left(t_{n_{1} 1}\right) & \ldots & Z_{1}\left(t_{n_{1} q}\right) \\
\vdots & \ddots & \vdots \\
Z_{n}\left(t_{n_{n} 1}\right) & \ldots & Z_{n}\left(t_{n_{n} q}\right)
\end{array}\right), \\
& \epsilon=\left(\begin{array}{c}
\left.\epsilon_{11}, \ldots, \epsilon_{1 n_{1}}, \ldots, \epsilon_{n n_{n}}\right)^{\tau}, \\
\end{array}\right)
\end{aligned}
$$

$D_{t}$

$$
=\left(\begin{array}{ccccc}
Z_{11} & \cdots & Z_{1 n_{1}} & \cdots & Z_{n n_{n}} \\
\frac{\left(t_{11}-t\right)}{h} Z_{11} & \cdots & \frac{\left(t_{1 n_{1}}-t\right)}{h} Z_{1 n_{1}} & \cdots & \frac{\left(t_{n n_{n}}-t\right)}{h} Z_{n n_{n}}
\end{array}\right)^{\tau} .
$$

Then, the solution to problem (6) is given by

$$
\begin{gathered}
\left(\widehat{a}_{1}(t), \ldots, \widehat{a}_{q}(t), h \widehat{b}_{1}(t), \ldots, h \widehat{b}_{q}(t)\right)^{\tau} \\
=\left(D_{t}^{\tau} \Omega_{t} D_{T}^{\tau}\right)^{-1} D_{t}^{\tau} \Omega_{t}\left(Y-X^{\tau} \beta\right) .
\end{gathered}
$$

Then, $\widehat{\theta}(t)$ can be given by

$$
\widehat{\theta}(t)=\left(I_{q}, 0_{q}\right)\left(D_{t}^{\tau} \Omega_{t} D_{T}^{\tau}\right)^{-1} D_{t}^{\tau} \Omega_{t}\left(Y-X^{\tau} \beta\right),
$$

where $I_{q}$ is $q \times q$ identity matrix and $0_{q}$ is $q \times q$ zero matrix. Denote

$$
\left(I_{q}, 0_{q}\right)\left(D_{t}^{\tau} \Omega_{t} D_{T}^{\tau}\right)^{-1} D_{t}^{\tau} \Omega_{t} \equiv\left(S_{11}(t), \ldots, S_{n n_{n}}(t)\right) ;
$$


then,

$$
\widehat{\theta}\left(t_{i j}\right)=\sum_{l=1}^{n} \sum_{m=1}^{n_{l}} S_{l m}\left(t_{i j}\right)\left(Y_{l m}-X_{l m}^{\tau} \beta\right)
$$

Substituting (12) into (4), we can obtain the approximate residuals as the following:

$$
\begin{aligned}
\widehat{r}_{i j}(\beta) & =Y_{i j}-X_{i j}^{\tau} \beta-Z_{i j}^{\tau} \sum_{l=1}^{n} \sum_{m=1}^{n_{l}} S_{l m}\left(t_{i j}\right)\left(Y_{l m}-X_{l m}^{\tau} \beta\right) \\
& =\widetilde{Y}_{i j}-\widetilde{X}_{i j}^{\tau} \beta
\end{aligned}
$$

where

$$
\begin{gathered}
\tilde{Y}_{i j}=Y_{i j}-Z_{i j}^{\tau} \sum_{l=1}^{n} \sum_{m=1}^{n_{l}} S_{l m}\left(t_{i j}\right) Y_{l m}, \\
\widetilde{X}_{i j}=X_{i j}-\left(\sum_{l=1}^{n} \sum_{m=1}^{n_{l}} S_{l m}\left(t_{i j}\right) X_{l m}\right)^{\tau} Z_{i j} .
\end{gathered}
$$

Similar to Owen [10], $\left\{\widehat{r}_{i j}(\beta), i=1, \ldots, n ; j=1, \ldots, n_{i}\right\}$ can be treated as a random sieve approximation of the random error sequence $\left\{\epsilon_{i j}, i=1, \ldots, n ; j=1, \ldots, n_{i}\right\}$. In order to deal with the correlation within group, we use the block empirical likelihood method. The block empirical likelihood procedure takes the "data" $\widehat{r}_{i j}(\beta), j=1, \ldots, n_{i}$ into account as a whole. Hence, similar to Xue and Zhu [13], we introduce the auxiliary random vector

$$
\widetilde{\eta}_{i}(\beta)=\int_{0}^{1} \widetilde{X}_{i}(t)\left[\widetilde{Y}_{i}(t)-\widetilde{X}_{i}^{\tau}(t) \beta\right] d N_{i}(t) .
$$

Following (13), if $\beta$ is true, then $E\left\{\widetilde{\eta}_{i}(\beta)\right\}=o(1)$. If one ignores the measurement error and replaces $X_{i j}$ by $W_{i j}$ in $\tilde{\eta}_{i}(\beta)$, one can show that the resulting estimator is inconsistent. As we all know, inconsistency caused by the measurement error can be overcome by applying the socalled correction for attenuation proposed by Fuller [22] in linear regression. With a similar way as in Zhao and Xue [14], the corrected-attenuation auxiliary vector is introduced and defined as

$$
\check{\eta}_{i}(\beta)=\int_{0}^{1}\left\{\widetilde{W}_{i}(t)\left(\widetilde{Y}_{i}(t)-\widetilde{W}_{i}^{\tau}(t) \beta\right)+\Sigma_{u u} \beta\right\} d N_{i}(t),
$$

where $\widetilde{W}_{i j}=W_{i j}-\left(\sum_{l=1}^{n} \sum_{m=1}^{n_{l}} S_{l m}\left(t_{i j}\right) W_{l m}\right)^{\tau} Z_{i j}$. The term $\Sigma_{u u} \beta$ aims to avoid the underestimating for the parameter caused by the measurement error. Therefore, the empirical likelihood ratio function for $\beta$ is defined as

$$
\mathscr{R}(\beta)=\max \left\{\prod_{i=1}^{n}\left(n p_{i}\right) \mid p_{i} \geq 0, \sum_{i=1}^{n} p_{i}=1, \sum_{i=1}^{n} p_{i} \check{\eta}_{i}(\beta)=0\right\} .
$$

A unique value for $\mathscr{R}(\beta)$ exists, provided that 0 is inside the convex hull of the point $\left(\check{\eta}_{1}(\beta), \ldots, \check{\eta}_{n}(\beta)\right)$. Using the Lagrange multiplier technique, the optimal value for $p_{i}$ is

$$
p_{i}=\frac{1}{n}\left\{1+\lambda^{\tau} \check{\eta}_{i}(\beta)\right\}^{-1}, \quad i=1, \ldots, n,
$$

where $\lambda=\left(\lambda_{1}, \ldots, \lambda_{n}\right)^{\tau}$ is the solution of the equation

$$
\frac{1}{n} \sum_{i=1}^{n} \frac{\check{\eta}_{i}(\beta)}{1+\lambda^{\tau} \check{\eta}_{i}(\beta)}=0
$$

Then, the block empirical log-likelihood ratio function is

$$
\mathscr{L} \mathscr{R}(\beta)=-2 \log \mathscr{R}(\beta)=2 \sum_{i=1}^{n} \log \left(1+\lambda^{\tau} \check{\eta}_{i}(\beta)\right) .
$$

In addition, by maximizing $\mathscr{L} \mathscr{R}(\beta)$, we can obtain the maximum empirical likelihood estimator (MELE) $\check{\beta}$. Let

$$
\check{\Gamma}=\frac{1}{n} \sum_{i=1}^{n} \int_{0}^{1}\left(\widetilde{W}_{i}(t) \widetilde{W}_{i}^{\tau}(t)-\Sigma_{u u}\right) d N_{i}(t) .
$$

If the matrix $\check{\Gamma}$ is invertible, then the MELE of $\beta$ can be given by

$$
\check{\beta}=\check{\Gamma}^{-1} \frac{1}{n} \sum_{i=1}^{n} \int_{0}^{1} \widetilde{W}_{i}(t) \widetilde{Y}_{i}(t)+o_{p}\left(n^{-1 / 2}\right) .
$$

According to $\check{\beta}$, we can define the estimator $\left\{\theta_{j}(\cdot), j=\right.$ $1, \ldots, q\}$ as

$$
\begin{aligned}
\check{\theta}(t) & =\left(\check{\theta}_{1}(t), \ldots, \check{\theta}_{q}(t)\right)^{\tau} \\
& =\left(I_{q}, 0_{q}\right)\left(D_{t}^{\tau} \Omega_{t} D_{T}^{\tau}\right)^{-1} D_{t}^{\tau} \Omega_{t}\left(Y-X^{\tau} \check{\beta}\right) .
\end{aligned}
$$

\section{Main Results}

To establish asymptotic properties of the block empirical loglikelihood ratio, we make the following assumptions. These assumptions are made by You and Zhou [4]. We use $\|\cdot\|$ to denote the Euclidean norm with $\|a\|=\left(a_{1}^{2}+\cdots+a_{n}^{2}\right)^{1 / 2}$ and $a=\left(a_{1}, \ldots, a_{n}\right)^{\tau}$.

Assumption 1. The random variable $t$ has a compact support $\Xi$. The density function $f(\cdot)$ of $t$ has a continuous second derivative and is uniformly bounded away from zero.

Assumption 2. The $p \times p$ matrix $E\left(X X^{\tau} \mid t\right)$ is nonsingular for each $t \in \Xi . E\left(X X^{\tau} \mid t\right), E\left(X X^{\tau} \mid t\right)^{-1}$, and $E\left(X Z^{\tau} \mid t\right)$ are all Lipschitz continuous.

Assumption 3. There is a $s>2$ such that $E\|X\|^{2 s}<\infty$, $E\|Z\|^{2 s}<\infty, E\|\epsilon\|^{2 s}<\infty$, and $E\|t\|^{2 s}<\infty$ and for some $\epsilon<2-s^{-1}$ such that $n^{2 \epsilon-1} h \rightarrow \infty$ as $n \rightarrow \infty$.

Assumption 4. $\alpha_{j}(\cdot), j=1, \ldots, q$ have the continuous second derivative in $t \in \Xi$.

Assumption 5. The kernel $K(\cdot)$ is a symmetric probability density function and is a bounded variation function on its support.

Assumption 6. The bandwidth $h$ satisfies $n h^{8} \rightarrow 0$ and $n h^{2} /(\log n)^{2} \rightarrow \infty$ as $n \rightarrow \infty$. 
The following theorem gives the asymptotic distribution of $\mathscr{L} \mathscr{R}(\beta)$.

Theorem 1. Assume that the Assumptions 1-6 hold; if $\beta$ is the true value of the parameter, then

$$
\mathscr{L} \mathscr{R}(\beta) \stackrel{\mathscr{D}}{\longrightarrow} \chi_{p}^{2} \text { as } n \longrightarrow \infty
$$

where $\stackrel{D}{\longrightarrow}$ denotes the convergence in distribution and $\chi_{p}^{2}$ is a chi-square distribution with $p$ degrees of freedom.

Then, we can construct the confidence regions for the parameter $\beta$. More precisely, for any $0<\alpha<1$, let $C_{\alpha}$ be such that $p\left(\chi_{p}^{2}>C_{\alpha}\right) \leq 1-\alpha$. Then,

$$
\mathscr{H}(\alpha)=\left\{\beta \in R^{p}: \mathscr{L} \mathscr{R}(\beta) \leq C_{\alpha}\right\}
$$

constitute a confidence region for $\beta$ with asymptotic coverage $1-\alpha$.

Theorem 2. Assume that the Assumptions 1-6 hold. Then, one has

$$
\sqrt{n}(\check{\beta}-\beta) \stackrel{\mathscr{D}}{\longrightarrow} N\left(0, \Gamma^{-1} \Sigma \Gamma^{-1}\right),
$$

where

$$
\begin{gathered}
\Gamma=E\left\{\int_{0}^{1}\left[W(t)-\mu_{t}^{\tau} Z(t)\right]^{\otimes 2} d N(t)\right\}, \\
B=E\left\{\int_{0}^{1}\left[W(t)-\mu_{t}^{\tau} Z(t)\right] \varepsilon(t)\right\}^{\otimes 2}, \\
A^{\otimes 2}=A A^{\tau}, \\
\mu(t)=E\left\{Z^{\tau}(t) W(t) \mid t\right\}^{-1} E\left\{Z(t) Z^{\tau}(t) \mid t\right\} .
\end{gathered}
$$

\section{Simulation Results}

In this section, we will conduct some simulations to the empirical likelihood (EL) method. The data are generated from

$$
\begin{aligned}
& y_{i}\left(t_{i j}\right)= x_{i}\left(t_{i j}\right) \beta+z_{1 i}\left(t_{i j}\right) \theta_{1}\left(t_{i j}\right) \\
&+z_{1 i}\left(t_{i j}\right) \theta_{2}\left(t_{i j}\right)+\epsilon_{i}\left(t_{i j}\right), \\
& w_{i}\left(t_{i j}\right)=x_{i}\left(t_{i j}\right)+u_{i}\left(t_{i j}\right),
\end{aligned}
$$

where $w_{i}(t) \sim N(0,1), \beta=1.5, z_{1 i}(t) \sim N(0,1), z_{2 i}(t) \sim$ $N(0,1), t \sim U(0,1), \theta_{1}(t)=\sin (2 \pi t), \theta_{2}(t)=\cos (2 \pi t), \epsilon_{i}(t) \sim$ $N(0,1), u_{i}(t)=b e_{i}\left(t_{j}\right)+e_{i}\left(t_{j-1}\right)$, and $e_{i}(t) \sim N(0,1)$.

In the simulation studies, for each combination of $n_{i}$, and $b$, we draw 1,000 random samples of sizes 100 or 200 from the above model, respectively. For each sample, a 95\% confidence interval for $\beta=1.5$ is computed using our block empirical likelihood method. The kernel function is taken as the Gauss kernel $K_{h}(t)=(1 / \sqrt{2 \pi} h) \exp \left(-(t)^{2} / 2 h^{2}\right)$. The "leave-onesample-out" method is used to select the bandwidth $h$. We define the score of $h$ as follows:

$$
\begin{gathered}
\mathrm{CV}(h)=\frac{1}{n} \sum_{i=1}^{n} \int_{0}^{1}\left\{Y_{i j}-W_{i j}^{\tau} \check{\beta}_{-i}-Z_{i j}^{\tau} \check{\theta}_{-i}\left(t_{i j}\right)\right\}^{2} \\
-\check{\beta}_{-i}^{\tau} \Sigma_{v v} \check{\beta}_{-i} d N_{i}(t) .
\end{gathered}
$$

Then cross-validation smoothing parameter $h_{\mathrm{CV}}$ is the minimizer of $\mathrm{CV}(h)$. Some representative coverage probabilities are reported in Table 1 .

\section{Proof of the Main Results}

In order to prove the main results, we first introduce several lemmas. Let $u_{k}=\int t^{k} K(t) d t, v_{k}=\int t^{k} K^{2}(t) d t, k=0,1,2,4$, $c_{n}=h^{2}+((\log n / n) h)^{1 / 2}, G(T)=E\left(Z Z^{\tau} \mid T\right), \Psi(T)=E\left(X Z^{\tau} \mid\right.$ $T)$, and $M=\left(Z_{1}^{\tau} \theta\left(t_{1}\right), \ldots, Z_{n}^{\tau} \theta\left(t_{n}\right)\right)^{\tau}$.

Lemma 3. Let $\left(X_{1}, Y_{1}\right), \ldots,\left(X_{n}, Y_{n}\right)$ be i.i.d random vector, where $Y_{i}$ is scalar random variable. Further, assume that $E\left|Y_{1}\right|^{s}<\infty, \sup _{x} \int|y|^{s} f(x, y) d y<\infty$, where $f(\cdot, \cdot)$ denotes the joint density of $(X, Y)$. Let $K(\cdot)$ be a bounded positive function with a bounded support, satisfying a Lipschitz condition. Given that $n^{2 \epsilon-1} h \rightarrow \infty$ for some $\epsilon<1-s^{-1}$, then size

$$
\begin{aligned}
\sup _{x} & \left|\frac{1}{n} \sum_{i=1}^{n}\left\{K_{h}\left(X_{i}-x\right) Y_{i}-E\left[K_{h}\left(X_{i}-x\right) Y_{i}\right]\right\}\right| \\
& =O_{p}\left(\left\{\frac{\log (1 / h)}{n h}\right\}^{1 / 2}\right) .
\end{aligned}
$$

Proof. This lemma can be found in Mack and Silverman [23].

Lemma 4. Let $\epsilon_{i}, i=1, \ldots, n$, be a sequence of multiindependent random variate with $E\left(\epsilon_{i}\right)=0$ and $E\left(\epsilon_{i}^{2}\right)<c<$ $\infty$. Then,

$$
\max _{1 \leq k \leq n}\left|\sum_{i=1}^{k} \epsilon_{i}\right|=O_{p}(\sqrt{n} \log n) .
$$

Further, let $\left(j_{1}, \ldots, j_{n}\right)$ be a permutation of $(1, \ldots, n)$. Then, one has

$$
\max _{1 \leq k \leq n}\left|\sum_{i=1}^{k} \epsilon_{j_{i}}\right|=O_{p}(\sqrt{n} \log n) .
$$

Proof. We can prove this lemma immediately by Kolmogorov inequality.

Lemma 5. Let $D_{1}, \ldots, D_{n}$ be i.i.d random variables. If $E\left|D_{i}\right|^{s}$ are uniformly bounded for $s>1$, then one has

$$
\max _{1 \leq i \leq n}\left|D_{i}\right|=o\left(n^{1 / s}\right) \text {. }
$$

Proof. This lemma can be found in Shi and Lau [24]. 
TABLE 1: Coverage probabilities (CP) and average lengths (AL) of the confidence intervals for $\beta=1.5$ and $\sigma^{2}=0.2$.

\begin{tabular}{|c|c|c|c|c|c|}
\hline \multirow{2}{*}{$k$} & \multirow{2}{*}{ Number of replicates } & \multicolumn{2}{|c|}{ CP (\%) } & \multicolumn{2}{|c|}{$\mathrm{AL}$} \\
\hline & & NA & EL & NA & EL \\
\hline \multicolumn{6}{|c|}{$b=0.3$} \\
\hline 100 & $n_{1}=\cdots=n_{50}=3$ & \multirow{2}{*}{93.27} & \multirow{2}{*}{93.34} & \multirow{2}{*}{0.2877} & \multirow{2}{*}{0.2789} \\
\hline & $n_{51}=\cdots=n_{100}=3$ & & & & \\
\hline \multirow{2}{*}{100} & $n_{1}=\cdots=n_{50}=3$ & \multirow{2}{*}{91.94} & \multirow{2}{*}{92.16} & \multirow{2}{*}{0.3079} & \multirow{2}{*}{0.3005} \\
\hline & $n_{51}=\cdots=n_{100}=2$ & & & & \\
\hline \multirow{2}{*}{200} & $n_{1}=\cdots=n_{100}=3$ & \multirow{2}{*}{93.98} & \multirow{2}{*}{94.26} & \multirow{2}{*}{0.2439} & \multirow{2}{*}{0.2432} \\
\hline & $n_{101}=\cdots=n_{200}=3$ & & & & \\
\hline \multirow{2}{*}{200} & $n_{1}=\cdots=n_{100}=3$ & \multirow{2}{*}{93.61} & \multirow{2}{*}{94.74} & \multirow{2}{*}{0.2651} & \multirow{2}{*}{0.2176} \\
\hline & $n_{101}=\cdots=n_{200}=2$ & & & & \\
\hline \multirow{3}{*}{100} & $b=0.6$ & \multirow{3}{*}{93.05} & \multirow{3}{*}{93.21} & & \\
\hline & $n_{1}=\cdots=n_{50}=3$ & & & \multirow{2}{*}{0.2967} & \multirow{2}{*}{0.2936} \\
\hline & $n_{51}=\cdots=n_{100}=3$ & & & & \\
\hline \multirow{2}{*}{100} & $n_{1}=\cdots=n_{50}=3$ & \multirow{2}{*}{91.74} & \multirow{2}{*}{91.86} & \multirow{2}{*}{0.3175} & \multirow{2}{*}{0.3114} \\
\hline & $n_{51}=\cdots=n_{100}=2$ & & & & \\
\hline \multirow[t]{2}{*}{200} & $n_{1}=\cdots=n_{100}=3$ & \multirow[t]{2}{*}{93.47} & 94.02 & 0.2711 & 0.2437 \\
\hline & $n_{101}=\cdots=n_{200}=3$ & & & & \\
\hline 200 & $n_{1}=\cdots=n_{100}=3$ & 92.82 & 93.69 & 0.2981 & 0.2646 \\
\hline 200 & $n_{101}=\cdots=n_{200}=2$ & 92.02 & 93.09 & 0.2901 & 0.2040 \\
\hline
\end{tabular}

Lemma 6. Suppose that Assumptions 1-6 hold; one has

$$
\begin{aligned}
& D_{t}^{\tau} \Omega_{t} D_{t}=N f(t) \Gamma \otimes G(t)\left(1+O_{p}\left(c_{n}\right)\right), \\
& D_{t}^{\tau} \Omega_{t} X_{t}=N f(t)(1,0)^{\tau} \otimes \Psi(t)\left(1+O_{p}\left(c_{n}\right)\right), \\
& D_{t}^{\tau} \Omega_{t} Z_{t}=N f(t)(1,0)^{\tau} \otimes G(t)\left(1+O_{p}\left(c_{n}\right)\right),
\end{aligned}
$$

which hold for all $t \in[a, b] \subset[0,1]$, where $\Gamma=\operatorname{diag}\left(1, u_{2}\right)$.

Proof. This follows immediately from the result that was obtained by Yang and $\mathrm{Li}[15]$.

Lemma 7. Suppose that Assumptions 1-6 hold; one has, when $t_{i j} \in[a, b]$,

$$
\begin{aligned}
S_{i}^{\tau}\left(t_{i j}\right) X & =Z_{i}^{\tau}\left(t_{i j}\right) G^{-1}\left(t_{i j}\right) \Psi\left(t_{i j}\right)\left(1+O_{p}\left(c_{n}\right)\right), \\
S_{i}^{\tau}\left(t_{i j}\right) M & =Z_{i}^{\tau}\left(t_{i j}\right) \theta\left(t_{i j}\right)\left(1+O_{p}\left(c_{n}\right)\right) .
\end{aligned}
$$

Proof. Let $S_{i}^{\tau}\left(t_{i j}\right)=\left[Z_{i}^{\tau}\left(t_{i j}\right), 0\right]\left(D_{t_{i j}}^{\tau} \Omega_{t_{i j}} D_{t_{i j}}\right)^{-1} D_{t_{i j}}^{\tau} \Omega_{t_{i j}}$; then, Lemma 7 can be directly attained by Lemma 6 .

Lemma 8. Suppose that the Assumptions 1-6 hold, one has

$$
\begin{aligned}
& \frac{1}{\sqrt{n}} \sum_{i=1}^{n} \check{\eta}_{i}(\beta) \stackrel{\mathscr{D}}{\longrightarrow} N(0, \Sigma), \\
& \frac{1}{n} \sum_{i=1}^{n} \check{\eta}_{i}(\beta) \check{\eta}_{i}^{\tau}(\beta) \stackrel{\mathscr{P}}{\longrightarrow} \Sigma, \\
& \max _{1 \leq x \leq n}\left\|\check{\eta}_{i}(\beta)\right\|=O_{p}\left(n^{1 / 2}\right),
\end{aligned}
$$

where $\Sigma$ is defined by (26).
Proof of Theorem 1. From (36), using the same arguments as were used in the proof of Owen [10], we have

$$
\|\lambda\|=O_{p}\left(n^{-1 / 2}\right),
$$

where $\lambda$ is defined in (19). Then, we have size

$$
\begin{aligned}
0 & =\frac{1}{n} \sum_{i=1}^{n} \frac{\check{\eta}(\beta)}{1+\lambda^{\tau} \check{\eta}_{i}(\beta)} \\
& =\frac{1}{n} \sum_{i=1}^{n} \check{\eta}_{i}(\beta)-\frac{1}{n} \sum_{i=1}^{n} \check{\eta}_{i}(\beta) \check{\eta}_{i}^{\tau}(\beta) \lambda+\frac{1}{n} \sum_{i=1}^{n} \frac{\check{\eta}_{i}(\beta)\left(\lambda^{\tau} \check{\eta}_{i}(\beta)\right)^{2}}{1+\lambda^{\tau} \check{\eta}_{i}(\beta)} .
\end{aligned}
$$

By using Lemma 8, we obtain

$$
\begin{gathered}
\sum_{i=1}^{n}\left(\lambda^{\tau} \check{\eta}_{i}(\beta)\right)^{2}=\sum_{i=1}^{n} \lambda^{\tau} \check{\eta}_{i}(\beta)+O_{p}(1), \\
\lambda=\left[\sum_{i=1}^{n} \check{\eta}_{i}(\beta) \check{\eta}_{i}^{\tau}(\beta)\right]^{-1} \sum_{i=1}^{n} \check{\eta}_{i}(\beta)+o_{p}\left(n^{-1 / 2}\right) .
\end{gathered}
$$

Applying the Taylor expansion to (20), we get that

$$
\mathscr{L} \mathscr{R}(\beta)=2 \sum_{i=1}^{n}\left[\lambda^{\tau} \check{\eta}_{i}(\beta)-\frac{1}{2}\left(\lambda^{\tau} \check{\eta}_{i}(\beta)\right)^{2}\right]+o_{p}(1) .
$$


Hence, together with (39), we have size

$$
\begin{aligned}
\mathscr{L} \mathscr{R}(\beta)= & {\left[\frac{1}{\sqrt{n}} \sum_{i=1}^{n} \check{\eta}_{i}(\beta)\right]^{\tau} } \\
& \times\left[\frac{1}{\sqrt{n}} \sum_{i=1}^{n} \check{\eta}_{i}(\beta) \check{\eta}_{i}^{\tau}(\beta)\right]^{-1}\left[\frac{1}{\sqrt{n}} \sum_{i=1}^{n} \check{\eta}_{i}(\beta)\right] \\
& +o_{p}(1) .
\end{aligned}
$$

Together with Lemma 8, this proves Theorem 1.

Proof of Theorem 2. Following the similar arguments as were used in the proof of Theorem 2 in Yang and Li [15], we have

$$
\check{\beta}-\beta=\check{\Gamma}^{-1} \frac{1}{n} \sum_{i=1}^{n} \check{\eta}_{i}(\beta)+o_{p}\left(n^{-1 / 2}\right) .
$$

By (35), we can prove $\check{\Gamma} \stackrel{\mathscr{P}}{\rightarrow} \Gamma$ by the law of large numbers. Together with Lemma 8 and Slutsky's theorem, this proves Theorem 2.

\section{References}

[1] W. Zhang, S.-Y. Lee, and X. Song, "Local polynomial fitting in semivarying coefficient model," Journal of Multivariate Analysis, vol. 82, no. 1, pp. 166-188, 2002.

[2] Q. Li, C. J. Huang, D. Li, and T.-T. Fu, "Semiparametric smooth coefficient models," Journal of Business \& Economic Statistics, vol. 20, no. 3, pp. 412-422, 2002.

[3] J. Fan and T. Huang, "Profile likelihood inferences on semiparametric varying-coefficient partially linear models," Bernoulli, vol. 11, no. 6, pp. 1031-1057, 2005.

[4] J. You and Y. Zhou, "Empirical likelihood for semiparametric varying-coefficient partially linear regression models," Statistics \& Probability Letters, vol. 76, no. 4, pp. 412-422, 2006.

[5] Z. Huang and R. Zhang, "Empirical likelihood for nonparametric parts in semiparametric varying-coefficient partially linear models," Statistics \& Probability Letters, vol. 79, no. 16, pp. 17981808, 2009.

[6] J. Fan, T. Huang, and R. Li, "Analysis of longitudinal data with semiparametric estimation of convariance function," Journal of the American Statistical Association, vol. 102, no. 478, pp. 632641, 2007.

[7] H. Liang, W. Härdle, and R. J. Carroll, "Estimation in a semiparametric partially linear errors-in-variables model," The Annals of Statistics, vol. 27, no. 5, pp. 1519-1535, 1999.

[8] H. Liang, S. W. Thurston, D. Ruppert, T. Apanasovich, and R. Hauser, "Additive partial linear models with measurement errors," Biometrika, vol. 95, no. 3, pp. 667-678, 2008.

[9] A. B. Owen, "Empirical likelihood ratio confidence intervals for a single functional," Biometrika, vol. 75, no. 2, pp. 237-249, 1988.

[10] A. Owen, "Empirical likelihood ratio confidence regions," The Annals of Statistics, vol. 18, no. 1, pp. 90-120, 1990.

[11] A. Owen, "Empirical likelihood for linear models," The Annals of Statistics, vol. 19, no. 4, pp. 1725-1747, 1991.

[12] E. D. Kolaczyk, "Empirical likelihood for generalized linear models," Statistica Sinica, vol. 4, no. 1, pp. 199-218, 1994.
[13] L. Xue and L. Zhu, "Empirical likelihood for a varying coefficient model with longitudinal data," Journal of the American Statistical Association, vol. 102, no. 478, pp. 642-654, 2007.

[14] P. Zhao and L. Xue, "Empirical likelihood inferences for semiparametric varying-coefficient partially linear errors-in-variables models with longitudinal data," Journal of Nonparametric Statistics, vol. 21, no. 7, pp. 907-923, 2009.

[15] H. Yang and T. Li, "Empirical likelihood for semiparametric varying coefficient partially linear models with longitudinal data," Statistics \& Probability Letters, vol. 80, no. 2, pp. 111-121, 2010.

[16] X. Hu, Z. Wang, and Z. Zhao, "Empirical likelihood for semiparametric varying-coefficient partially linear errors-in-variables models," Statistics \& Probability Letters, vol. 79, no. 8, pp. 10441052, 2009.

[17] X. Wang, G. Li, and L. Lin, "Empirical likelihood inference for semi-parametric varying-coefficient partially linear EV models," Metrika, vol. 73, no. 2, pp. 171-185, 2011.

[18] G.-L. Fan, H.-X. Xu, and H.-Y. Liang, "Empirical likelihood inference for partially time-varying coefficient errorsin-variables models," Electronic Journal of Statistics, vol. 6, pp. 1040-1058, 2012.

[19] G.-L. Fan, H.-Y. Liang, and J.-F. Wang, "Statistical inference for partially time-varying coefficient errors-in-variables models," Journal of Statistical Planning and Inference, vol. 143, no. 3, pp. 505-519, 2013.

[20] J. You, G. Chen, and Y. Zhou, "Block empirical likelihood for longitudinal partially linear regression models," The Canadian Journal of Statistics, vol. 34, no. 1, pp. 79-96, 2006.

[21] X. Hu, "The block empirical likelihood method of the semivarying coefficient model with application to longitudinal data," Communications in Statistics. Theory and Methods, vol. 40, no. 8, pp. 1342-1351, 2011.

[22] W. A. Fuller, Measurement Error Models, John Wiley \& Sons, New York, NY, USA, 1987.

[23] Y. P. Mack and B. W. Silverman, "Weak and strong uniform consistency of kernel regression estimates," Zeitschrift für Wahrscheinlichkeitstheorie und Verwandte Gebiete, vol. 61, no. 3, pp. 405-415, 1982.

[24] J. Shi and T.-S. Lau, "Empirical likelihood for partially linear models," Journal of Multivariate Analysis, vol. 72, no. 1, pp. 132148, 2000. 


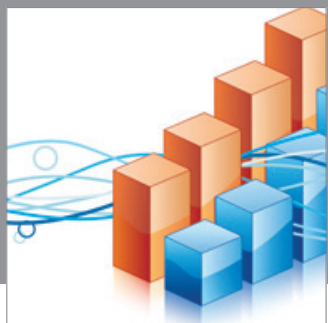

Advances in

Operations Research

mansans

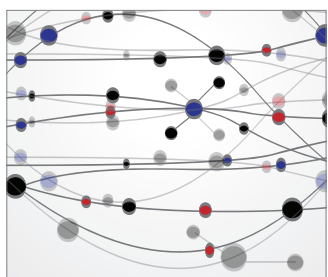

The Scientific World Journal
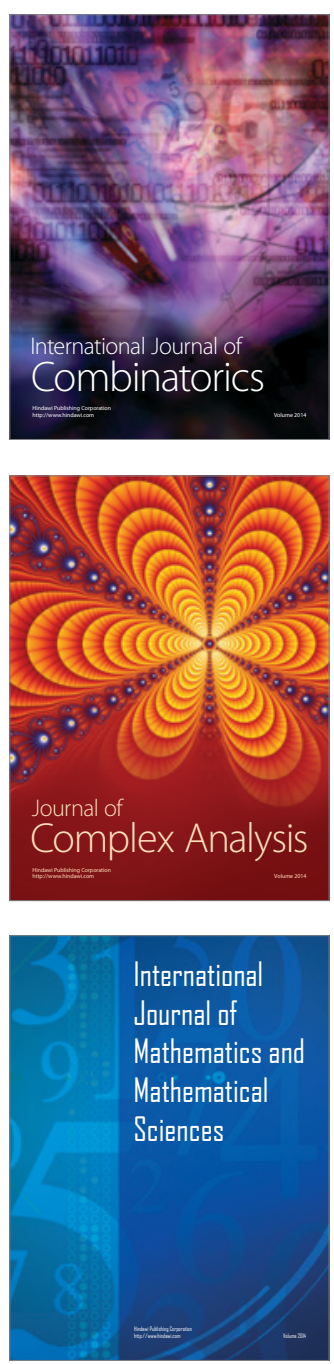
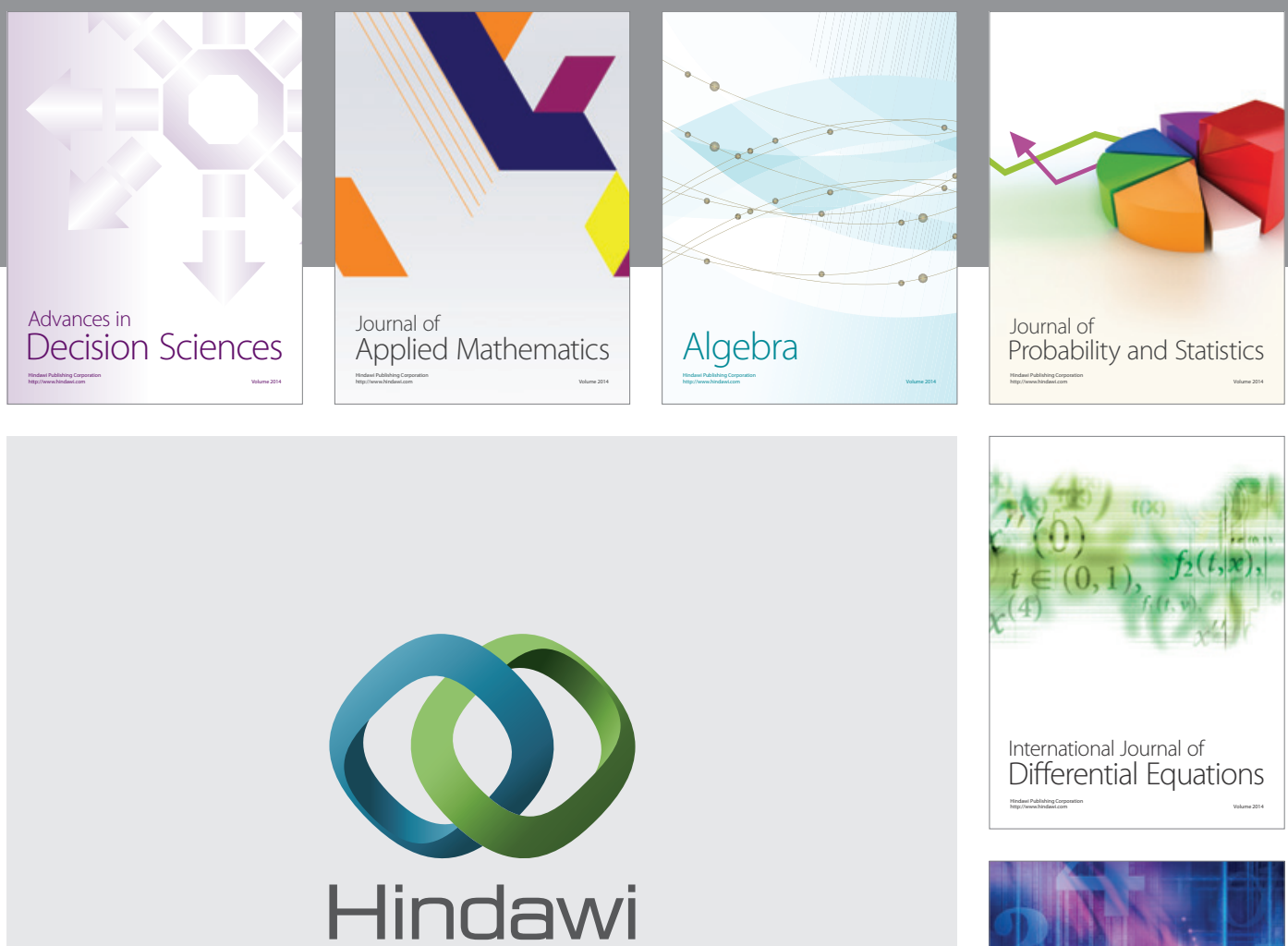

Submit your manuscripts at http://www.hindawi.com
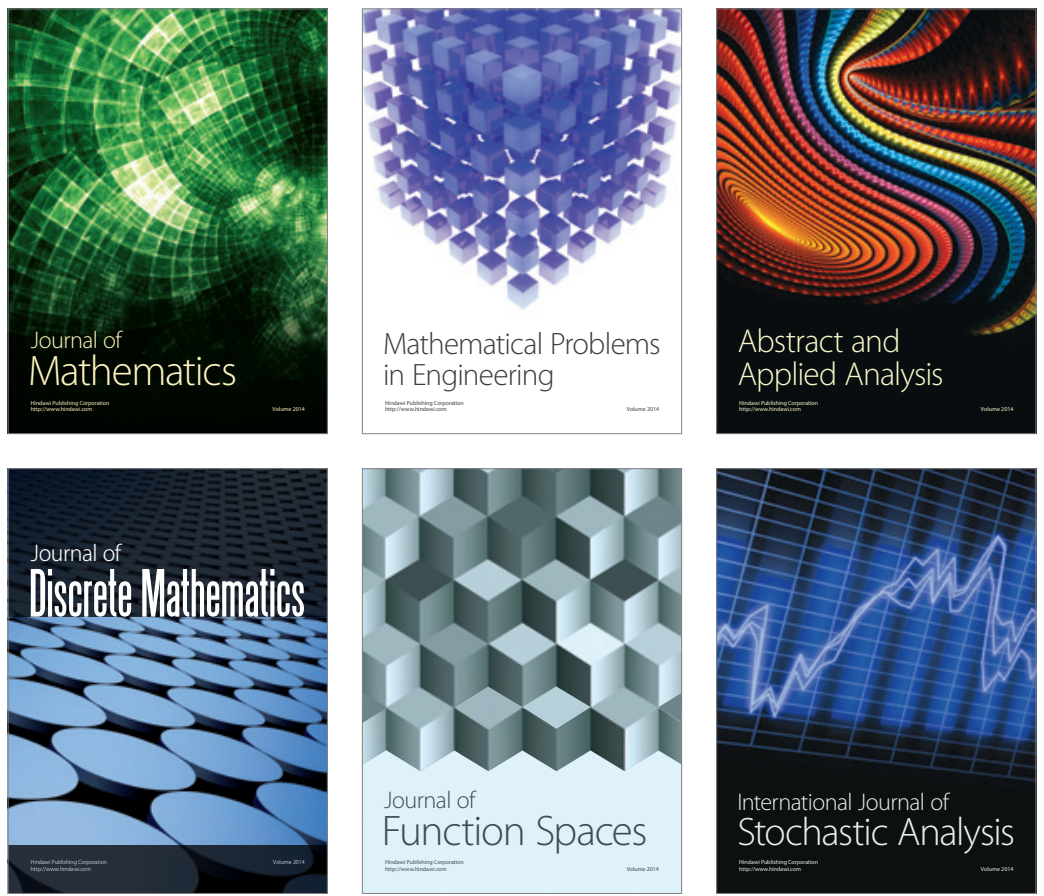

Journal of

Function Spaces

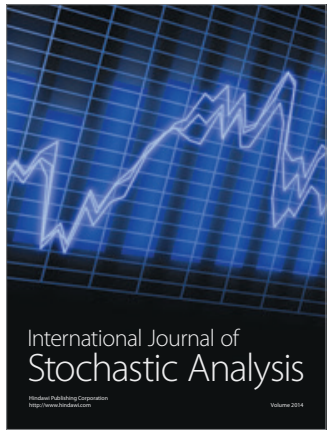

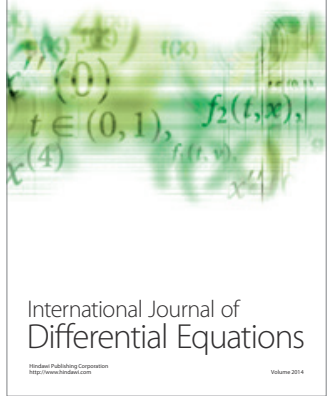
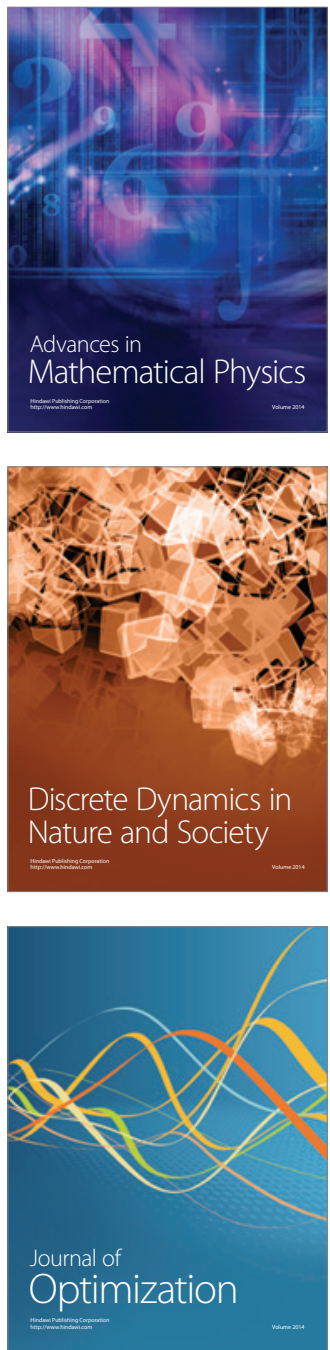